\title{
Adaptation of coordination mechanisms to network structures
}

\author{
Herwig Mittermayer; Carlos Rodríguez Monroy \\ Universidad Politécnica de Madrid (SPAIN) \\ h.mittermayer@web.de; crmonroy@etsii.upm.es
}

Received October 2008

Accepted November 2008

Abstract: The coordination efficiency of Supply Chain Management is determined by two opposite poles: benefit from improved planning results and associated coordination cost. The centralization grade, applied coordination mechanisms and IT support have influence on both categories. Therefore three reference types are developed and subsequently detailed in business process models for different network structures. In a simulation study the performance of these organization forms are compared in a process plant network. Coordination benefit is observed if the planning mode is altered by means of a demand planning IT tool. Coordination cost is divided into structural and activity-dependent cost. The activity level rises when reactive planning iterations become necessary as a consequence of inconsistencies among planning levels. Some characteristic influence factors are considered to be a reason for uninfeasible planning. In this study the effect of capacity availability and stochastic machine downtimes is investigated in an uncertain demand situation. Results that if the network runs with high overcapacity, central planning is less likely to increase benefit enough to outweigh associated cost. Otherwise, if capacity constraints are crucial, a central planning mode is recommendable. When also unforeseen machine downtimes are low, the use of sophisticated IT tools is most profitable.

Keywords: coordination mechanisms, distributed decision making, network configuration 


\section{Introduction}

Due to the increasing worldwide allocation of manufacturing processes, the value creation process gets continuously more divided and dispersed as well. Therefore the efficient coordination of information and material flow gained an outstanding importance to better meet customer demands and cut operational costs. Enterprise Resource Planning (ERP) systems became the major backbone technology for the execution of business processes, but contrarily to the name's indication it does not provide wide planning functionalities. Based on those principally transactionoriented systems, the managers either applied basic decision rules or extracted data and developed their own decision support systems in spreadsheets. To provide a common supply-chain-wide decision tool, in the mid 90's, modern ERP vendors started to offer Advanced Planning Systems (APS). The aim of those addon tools is to reduce organization expenses and improve planning results, thus better achieve production and logistics objectives. Central feature of APS are optimization methods that overcome the inconvenience of ERP planning methods by simultaneously considering material, resource and time constraints. Up to now success was mostly limited to those multinational supply networks from automotive, electronic and food industry. Generally speaking, success factors have been the implementation of economies of scale, expedient structural characteristics of those industry environments and the powerful position as supply chain leader to enforce the new business model across the whole supply chain. Under perfect conditions, the improvement of planning results outweighs clearly the associated organization cost. If conditions are less favourable, the organization cost constitutes an important expense that in some cases does not justify the investment.

\section{Literature review}

Driven by costly implementation fiascos, research has started to investigate the reason why it was not possible to realize the benefits from software-inherent optimization models. On the one hand the deterministic programming does not contemplate the real world uncertainty, thus expected optimized planning cannot be realized. Gupta and Maranas (2003) increase planning quality by incorporating uncertain demand and obtain improved expected values for service level and cost. On the other hand "many logistics-based approaches to SCM are still within the traditional realm of one central DMU [Decision Making Unit]. The same holds just 
as true for most of the existing supply chain software" (Schneeweiss \& Zimmer, 2004). As a consequence some recent research sheds light on alternative planning modes, e.g. concerning:

- Comparison of collaborative coordination mechanism against a pure hierarchical planning approach (Schneeweiss \& Zimmer, 2004; Li \& Wang, 2007) or in distinctive organization structures (Meijboom \& Obel, 2007),

- Consideration of coordination costs complementary to benefits from improvement in planning and control (Kim \& Park, 2008),

- Analysis of network attributes that affect the supply chain performance (Garavelli, 2003; Beamon \& Chen, 2001).

Little research is carried out on how network properties affect the coordination efficiency. Furthermore, most known models do not explicitly incorporate coordination cost although benefits from improved planning might be significantly reduced by coordination cost. The frequency of iterative planning procedures on the one hand and beneficial effects on the other depend to a large extend on dynamic parameters. Therefore, in this investigation stochastic simulation is employed to back up the situation-dependent selection of efficient organization types.

\section{Evaluating structure-specific coordination forms}

In this paper alternative coordination forms - with and without the use of additional IT - are developed for industrial networks (Figure 1). The derived process-oriented flow models are generic for a basic network type, but the coordination efficiency depends on influence factors that describe the network configuration. The planning process is valued by means of coordination benefit and cost for each one of the predefined coordination forms. Coordination benefit is mainly achieved by improving manufacturing and logistics performance (capacity utilization, lead time reduction, lower inventories, etc.) while coordination cost is influenced by information flow and processing or resource assignment conditioned by organization and IT support.

Exemplarily, for two distinct manufacturing structures the coordination forms are characterized and detailed in process-oriented models, individually for each 
coordination form. It turns out that in the distinct network structures fundamentally different task dependencies prevail. In a simulation study the coordination forms are compared by quantifying coordination benefit and cost for one of the network structures.

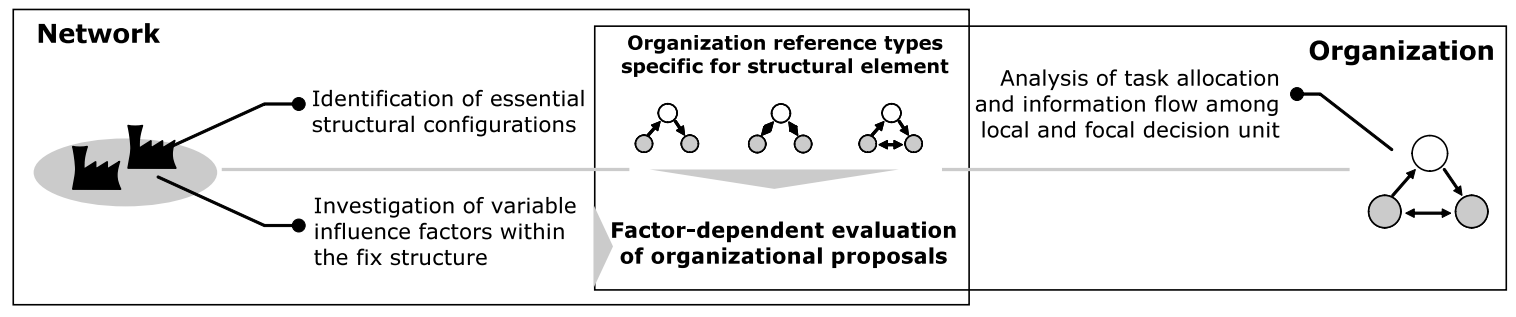

Figure 1. "Process to derive appropriate coordination mechanisms from network characteristics".

\section{Multi-plant strategies}

A widely accepted classification summarizes four important multi-plant configurations among Fortune 500 companies in the USA (Schmenner, 1982). If a product plant strategy is pursued, each plant is assigned to manufacture one product for the entire global market. In contrast, under a market area plant strategy, each plant supplies almost the entire line of products to a dedicated geographical market (Garavelli, 2003). In order to benefit from economies of scale by focusing on a process plant strategy, the manufacturing process is split and specific production stages are assigned to individual plants. The downstream plant is supplying all markets. Alternatively, the general-purpose plant strategy maintains flexible plants that are capable of manufacturing the whole product programme and distribute it to any market.

This paper focuses on two of the four mentioned configurations, namely the highly specialized process and the flexible general-purpose plant strategy.

\section{Coordination forms}

The running of a formalized central organization structure implies extra costs that are independent from the activity-level. These emerge because a central department has to be added to the two existing local organizations. Therefore the installation of a superior organization unit means additional personnel cost such as wages, but also additional organization cost for all supporting departments, like 
Human Resources, Finance, etc. Coordination mechanisms are applied to assure the information flow among departments.

\subsection{Coordination mechanism}

The more complex the planning environments the more specialized are the activities and the more interfaces exist among departments. Different mechanisms can be used to link the planning tasks. The resulting set of mechanisms within the structural organization here is defined as the coordination form. In the following different coordination mechanisms are described.

\section{Planning}

The aim of a planning procedure is to achieve decisions about future activities. Generally, the procedure may be split into the steps of generation of alternatives, their evaluation and adaptation, and the final selection of the best plan (Schotten, 1998). In the field of Supply Chain Management, typically a standard procedure is distinguished from a reactive one. In a standard routine an initial plan is elaborated. If inconsistencies occur on lower levels or if new information forces to start iteration, this planning procedure is called reactive.

\section{Horizontal and vertical communication}

Communication may be applied uni- or bi-directionally. Horizontal communication is used to facilitate information to units that are located on the same planning level within the hierarchy. If each unit focuses on its objectives the relationship is competitive; otherwise if both units pursue common goals, it is a collaborative relationship. In contrast vertical communication is the directed top-down flow of either instructions or targets. Instructions constitute definitive inputs to the baselevel, while targets consist of rough cut plans elaborated by the top-level. On the base-level they have to be considered although they are not strictly binding. If important constraints are violated, a bottom-up backflow of a proposal may be induced. This procedure is repeated until a feasible solution is found.

\section{$\underline{\text { Heuristics }}$}

Heuristics are rules of thumb that support the standardization of activities and communication leading to shorten processing times. Therefore their implementation is appropriate to reduce coordination cost if the reduction in 
processing cost outweighs the expense for set-up and continuous adjustment of those rules and the loss of flexibility due to programming. In the context of SCM, for example a standardized allocation heuristic such as a fixed product-plant assignation rule avoids further coordination efforts, but eliminates opportunities for improved planning results. Usually this mechanism limits the decision autonomy of the lower level units due to its binding character.

\section{Planning models}

In the following section interdependencies among planning tasks on tactical and operational level are represented. The strategic level is not incorporated because strategic decisions have been taken and are reflected by one of the four multi-plant strategies. As indicated before, this includes decisions about principal product-plant assignments, the logistic channel and markets selection (Roesgen \& Schuh, 2005). In the following three different coordination forms (Collaborative, Distributed or Hierarchical Planning) are presented. They can be combined with any one of the strategic configurations, although this paper is limited to the process and general purpose plant strategy.

\subsection{Process plant strategy}

The traditional purchaser-supplier material flow takes place if a process plant strategy is pursued. Then decisions considering distinctive planning periods are interdependent, because the supplier manufactures the required goods in earlier periods. In a Collaborative Planning environment planning activities are entirely decentralized. There is no central decision unit. The downstream located plant is distributing to the markets and therefore should realize the demand planning (Figure 2). The period-specific demands enter in the production planning. In the next step the MRP determines the components and material requirements netting existing stocks and reserved inventories. Then a rough cut order sequence is generated taking into account economic lot-sizing. Finally the scheduling determines the short term planning on the operational level. Inconsistencies may occur on any level and result in a feedback-flow (dotted line) to upper levels and the partial repetition of activities. The collaborative planning mode assumes that apart from short term orders, mid-term production planning data is provided to the upstream located plant. The supplier is running an equal top-down planning procedure and acknowledges the purchaser's plan - or denies it providing a 
modified feasible plan. Collaborative Planning includes data exchange or mutual access to ERP databases.

Central planning implies always the existence of a central, superior decision unit. Nevertheless, different degrees of centralization exist. In a Distributed Planning environment, the central unit elaborates a rough cut network production plan. Subsequent activities are executed by the local unit. If important constraints are violated an iterative planning loop has to be started.

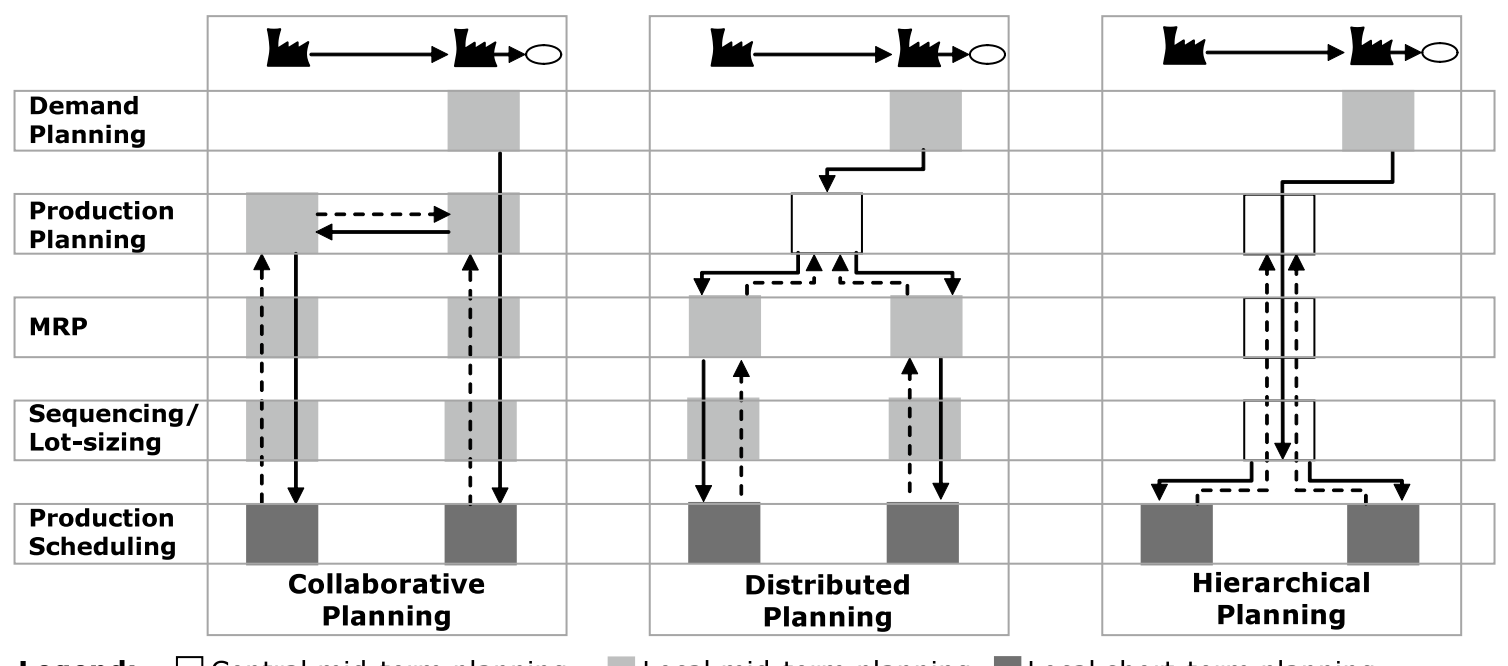

Legend: $\square$ Central mid-term planning Local mid-term planning Local short-term planning

Figure 2. "Local and central planning under a process plant strategy".

In a Hierarchical Planning procedure, the locally realized demand planning is followed by the central execution of all subsequent mid-term tasks. Some or all of the activities are supported by means of APS. Major property of those systems is the consideration of cross-plant interdependencies among routings and bills-ofmaterials. The local plant management is supposed to decompose the data and respect the binding instructions. Due to the high planning precision, rarely iterative procedures are foreseen so that output data mainly forms direct input to the local scheduling. For example, a production optimization tool avoids unfeasible plans, because time, capacity and material constraints would be respected simultaneously. From an organizational perspective the mainly unidirectional topdown information flow avoids time-consuming and costly iterative procedures. Nevertheless, partial implementations are possible. This means that only one or 
some modules from an APS package may be installed. Then iterative procedures are observable.

\subsection{General-purpose plant strategy}

In contrast to the process plant strategy, in a general-purpose plant configuration all facilities manufacture an equal product portfolio and supply the same markets. In this case, interdependencies occur among equal planning periods. Furthermore, the main coordination issue is not the sequential material flow, but the allocation of orders to facilities. Therefore coordinated planning is supposed to be initiated earlier, on the demand planning level (Figure 3). While the Collaborative Planning on the lower levels is similar to the procedure pursued in a process plant network, Distributed Planning is organized distinctively. Due to the strong interdependencies of demand and production planning, both activities are realized by the central unit. The MRP is run for the whole network because similarities in material requirements are probable and economies of scales are likely to be realizable by bundling purchase orders.

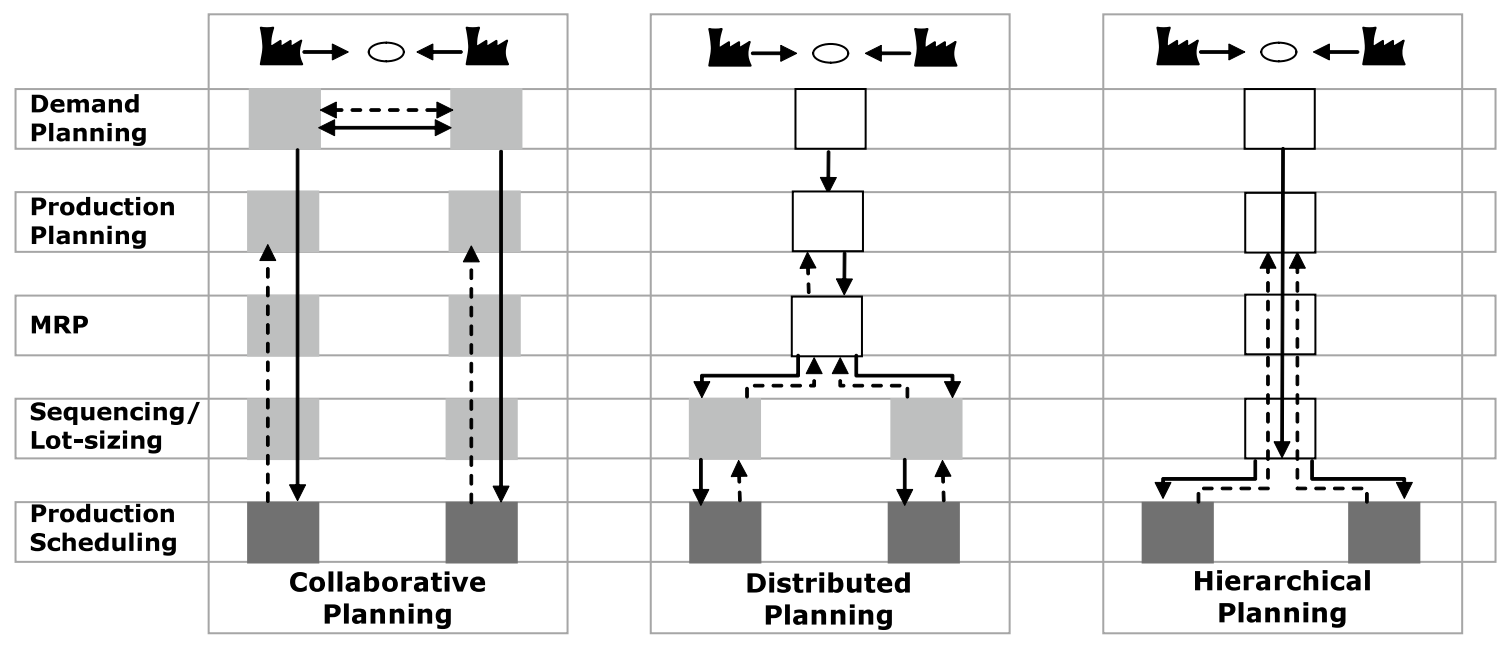

Legend: $\square$ Central mid-term planning Local mid-term planning Local short-term planning

Figure 3. "Local and central planning under a general-purpose plant strategy".

\section{Coordination efficiency and influence factors}

In order to reduce the complexity in SCM some simplifications of the planning task are usually applied. This may lead to contradictions that have to be solved. Time, material and capacity constraints are not considered simultaneously in the traditional MRP logic. The manifestation of inconsistencies on lower levels makes 
iterations necessary to achieve feasible plans. By means of APS those constraints are incorporated at a mid-term horizon. Data decomposition from rough to fine planning levels may cause unrealistic planning as well. APS tools allow the modelling of combined product-process segments so that material and process information is aggregated jointly. In order to reduce coordination costs caused by iterations among different hierarchical levels, traditional ERP systems are updated with low frequency (cyclical overnight batch-processing). The increased data precision level supported by APS reduces the need for iterations. In same cases even event-oriented up-dating in case of unforeseen plan deviations is becoming economically feasible. Important deviations are consequences of unforeseen events that have to be answered with reactive planning (Schotten, 1998). Those, for example, are input failures (e.g. stock-out of raw material), process failures (e.g. machine breakdown) or output failures (e.g. sales forecast error).

The coordination efficiency is determined by two concepts. On the one hand, improving planning procedure reduces stocks, increases the capacity utilisation, and shortens lead times, etc. The better achievement of manufacturing and logistics objectives is quantified by total revenue; penalties for lost sales may be incorporated to represent the long-term effect of decreasing customer retention. On the other hand, the establishment of organizational structures and IT support requires up-front investments and continuous expenses to run the organization. In what follows we distinguish between structural and activity-dependent organization cost and structural technology cost. The activity-dependent cost is charged proportionally to the number of (iterative) planning procedures. It is a function of employees involved, personnel unit cost, frequency and processing time and differs substantially for the regarded planning modes. The structural cost rates for technology correspond to hardware and software, employee's training or consulting concerning implementation and reorganization. The structural organization cost rates refer to continuous expenses, as a consequence of the installation of a central planning unit.

Influence factors are those parameters that have important impact on the coordination efficiency. Each planning tasks has different input and output variables or parameters. Therefore some influence factor might have a deep impact on the outcome of one activity while for other tasks it might be irrelevant. The use of costly sophisticated planning software is only recommendable if favourable production network characteristics prevail. For example, the benefit from an 
optimized production plan varies widely: If capacity utilization is high, the violation of constraints is probable and a highly beneficial optimization process is initiated, when a corresponding system is installed. Generally, the most important benefits of APS on business performance are expected when the level of variability is high and reactive procedures are probable as adequate answer to emerging exceptions (Stadtler \& Kilger, 2000). If conditions rarely change, the problem can be solved satisfactorily by simple planning heuristics instead of implementing costly IT systems.

The following simulation study focuses on the possible application of a demand planning module, and the influence of some selected factors on the coordination efficiency for the presented coordination forms under the conditions of a process plant strategy.

\section{Simulation study}

In the current study a simple two-plant process strategy is pursued. A single product type is manufactured; based on a single component, which is produced with a 1-month lead time in the upstream facility. Transport is not incorporated in the model, thus transport time is zero. The simulation considers a two-period sales planning horizon. Demand is uncertain; in all simulation runs a uniform distribution is assumed with its limit at $+/-5 \%$ of the mean. The demand planning department elaborates sales forecasts for each of the two months with different forecast accuracy, the first being twice as precise as the second. The demand is reported to the production planning unit. Taken into account information about free capacity, the quantities are allocated to the periods. Components and products that cannot be manufactured in the corresponding period are delayed or anticipated, respectively. Then subsequent activities (MRP, etc.) are realized. When the manufacturing orders are handed over to the local scheduling level, stochastic machine downtime is detected. As a consequence, if capacity constraints are violated, an iterative planning procedure has to be executed. One simulation run includes 600 runs (incl. one iterative planning procedure if necessary); each run equals a 2-month period.

Figure 4 illustrates the four simulation setups that are tested for the three coordination forms: Collaborative, Distributed and Hierarchical Planning. The latter 
coordination form implies the use of a demand planning tool that reduces the forecast error by $50 \%$.
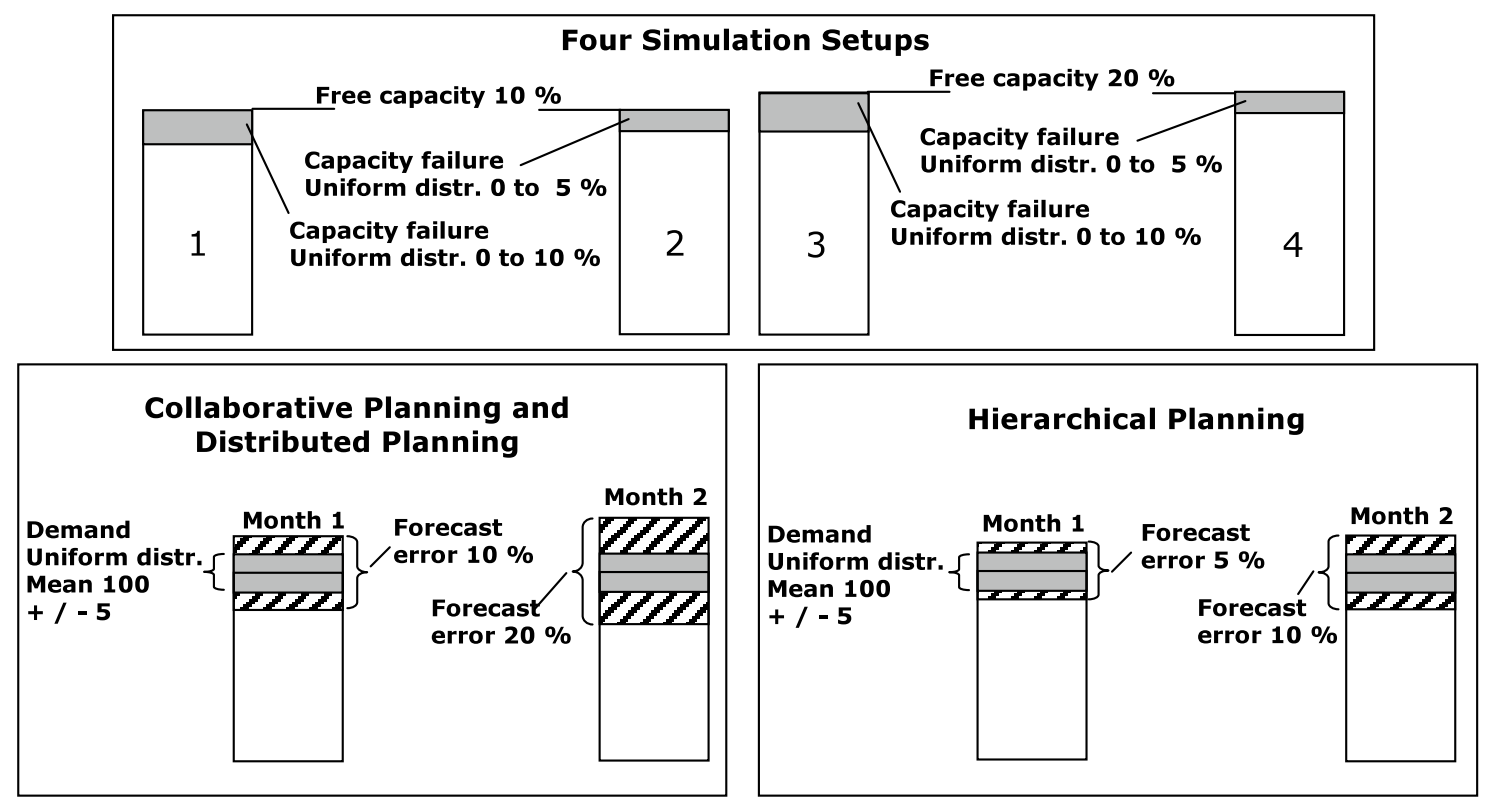

Figure 4. "General simulation set-ups for coordination forms (demand in 1.000 units)".

The coordination benefit is measured as follows: The production quantities are allocated on the basis of the reported demand. This figure does not equal the real demand due to the modelled forecast error. Stock cost is considered if production exceeds demand. Two different types of penalties are incorporated: Late delivery due to delayed production (one period) leads to a $10 \%$ penalty on the margin. Product stock-outs are weakening the competitive position; thus lost margins are charged as penalties.

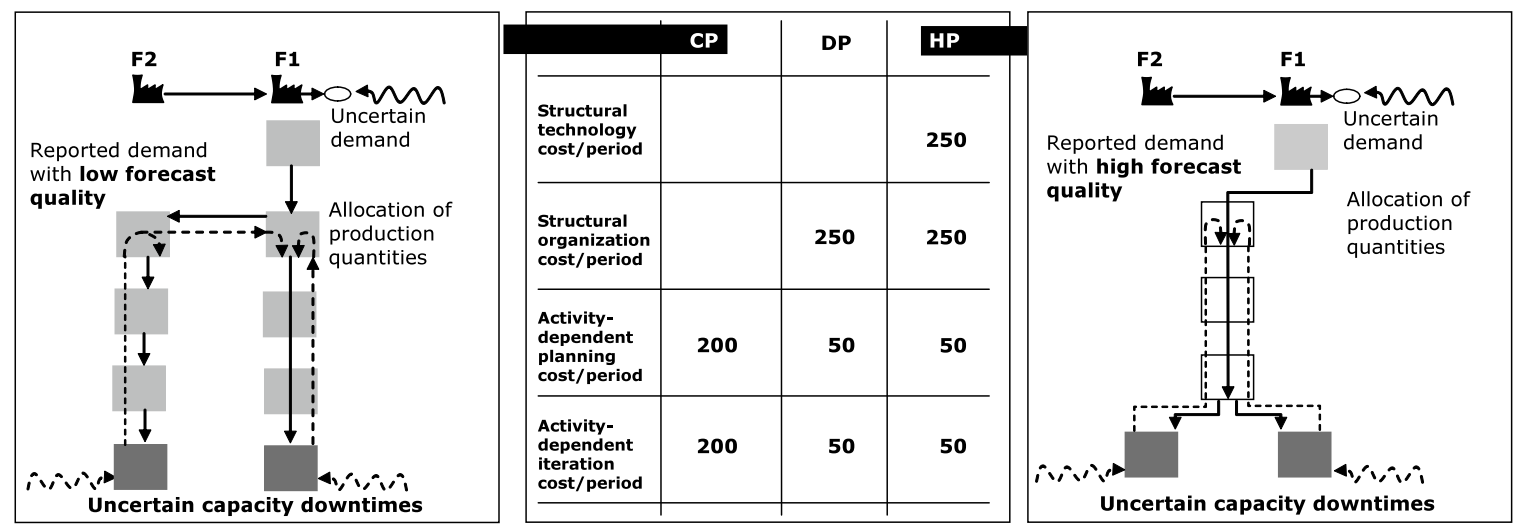

Figure 5. "Information flow schemes and resulting coordination cost [1.000 € / 2-periods]". 
An important issue in this investigation is the explicit consideration of coordination costs. Figure 5 illustrates two coordination forms, Collaborative Planning (CP) and Hierarchical Planning (HP) constituting opposite poles. The decentralized CP does not require additional central organization structures; therefore no structural organization cost rate is listed. Nevertheless, the iterative planning procedure (if necessary) causes a complex and costly coordination process and therefore is valued with a relatively high cost rate. The intermediate stage of DP results in extra structural organization cost, but no extra structural technology cost. The planning procedure is exactly the same in the cases of CP and DP, thus differences concerning the coordination efficiency solely are due to different coordination costs. In the case of HP it is assumed that a demand planning module is installed. This means that on the one hand extra cost (structural technology cost) have to be incorporated, but on the other hand the forecasting procedure is improved and delivers additional coordination benefit.

The coordination benefit is defined as:

- Sales revenues,

- Minus production and storage costs,

- Minus penalty cost for late delivery and for lost sales.

Coordination cost is the sum of:

- Structural organization cost,

- Structural technology cost,

- Activity-dependent planning cost,

- Activity-dependent iteration cost.

Coordination efficiency is coordination benefit minus coordination cost.

The simulation study is carried out using the student version of the spreadsheet simulation software Crystal Ball 2000. For each one of the 600 runs, the following steps have been performed:

- Generate random variables for demand and machine failure 
- Allocate production quantities to periods, i.e. production quantities can be anticipated or delayed to balance capacity loading of two periods.

- Calculate coordination benefit and cost

The objective of the present simulation study is to point out the causal relationship between influence factors and coordination efficiency for different organization forms. There is a broad spectrum of possible influence factors; in this case two factors have been studied: free capacity and machine downtimes. Each organization form is characterized by further individual properties such as organization cost (see Figure 5) and forecast errors (see Figure 6). Apart, various parameters are remained constant throughout the simulation series for all organization forms; the most important are:

- Sales per unit: $156 €$

- Production cost per unit, finished product: $100 €$

- Production cost per unit, component: $20 €$

- Storage cost per unit and period, finished product: $3 €$

- Storage cost per unit and period, component: $1 €$

Values are adapted from an industrial case in order to reflect real world proportions.

\section{Results and conclusions}

Table 1 summarizes the results gained from four different simulation series.

\begin{tabular}{|c|c|c|c|c|c|c|c|c|c|c|c|c|}
\hline \multirow[b]{3}{*}{$\begin{array}{l}\text { Fix free } \\
\text { capacity }\end{array}$} & \multirow[b]{3}{*}{$\begin{array}{l}\text { Mean Cap. } \\
\text { Downtime }\end{array}$} & \multirow{2}{*}{\multicolumn{2}{|c|}{$\begin{array}{c}\text { No. of iterative } \\
\text { plannings (based on } \\
600 \text { runs each) }\end{array}$}} & \multirow{2}{*}{\multicolumn{3}{|c|}{\begin{tabular}{|c|} 
Collaborative Planning \\
Coordination ...
\end{tabular}}} & \multirow{2}{*}{\multicolumn{3}{|c|}{$\frac{\text { Distributed Planning }}{\text { Coordination ... }}$}} & \multirow{2}{*}{\multicolumn{3}{|c|}{$\frac{\text { Hierarchical Planning }}{\text { Coordination ... }}$}} \\
\hline & & & & & & & & & & & & \\
\hline & & $\begin{array}{c}\mathrm{CP} \text { and } \\
\text { DP }\end{array}$ & HP & $\begin{array}{c}\text { Effi- } \\
\text { ciency }\end{array}$ & Benefit & Cost & $\begin{array}{c}\text { Effi- } \\
\text { ciency }\end{array}$ & Benefit & Cost & $\begin{array}{c}\text { Effi- } \\
\text { ciency }\end{array}$ & Benefit & Cost \\
\hline 10 & 10 & 443 & 374 & 4 & & 348 & 25 & & 337 & 6.142 & & 581 \\
\hline 1 & & 41 & 251 & & & 337 & & & 334 & & & 571 \\
\hline $20 \%$ & $10 \%$ & 229 & 14 & 6.086 & 6.362 & 276 & 6.043 & 6.362 & 319 & 6.172 & 6.723 & 551 \\
\hline $20 \%$ & $5 \%$ & 111 & 0 & 6.047 & 6.284 & 237 & 5.975 & 6.284 & 309 & 6.173 & 6.723 & 550 \\
\hline
\end{tabular}

Table 1. "Simulation results [mean values in $1.000 € / 2$-periods]". 
The coordination efficiency (benefit minus cost) is the overall indicator to evaluate the coordination forms. Figure 6 depicts the most important relationships. The coordination cost ratio of CP to DP and the coordination efficiency delta of HP to CP are represented (Figure 6).

There is no difference in coordination benefit comparing CP and DP. This is because with both the same planning method is applied. Hence, by focusing on the coordination cost all differences in coordination efficiency can be explained. The coordination cost of CP relative to those incurred with DP are at its lowest point if machine failure is relatively low and if the situation is characterized by a high level of free capacities (series 4). For higher, less predictable downtimes the DP type performs better. In general, with capacity constraints becoming eminent, the number of iterations and therefore the activity-dependent cost forces up the coordination cost. Thus, in the most relaxed case of high free capacity and low downtimes CP significantly outperforms the DP type. If coordination efficiency of $\mathrm{HP}$ is compared to DP, in all simulation runs the HP type achieves better results. Nevertheless, the highest relative performance of HP assumes low machine failures and limited capacities (series 2). This is because on the one hand improvement from demand planning cannot be realized if on the short-term level important uncertainties exist. Then the additional expense for the elaboration of more precise sales forecasts lead to production plans that are not feasible. On the other hand, a precise forecast is more valuable, if the capacities are constrained on the mid-term level.

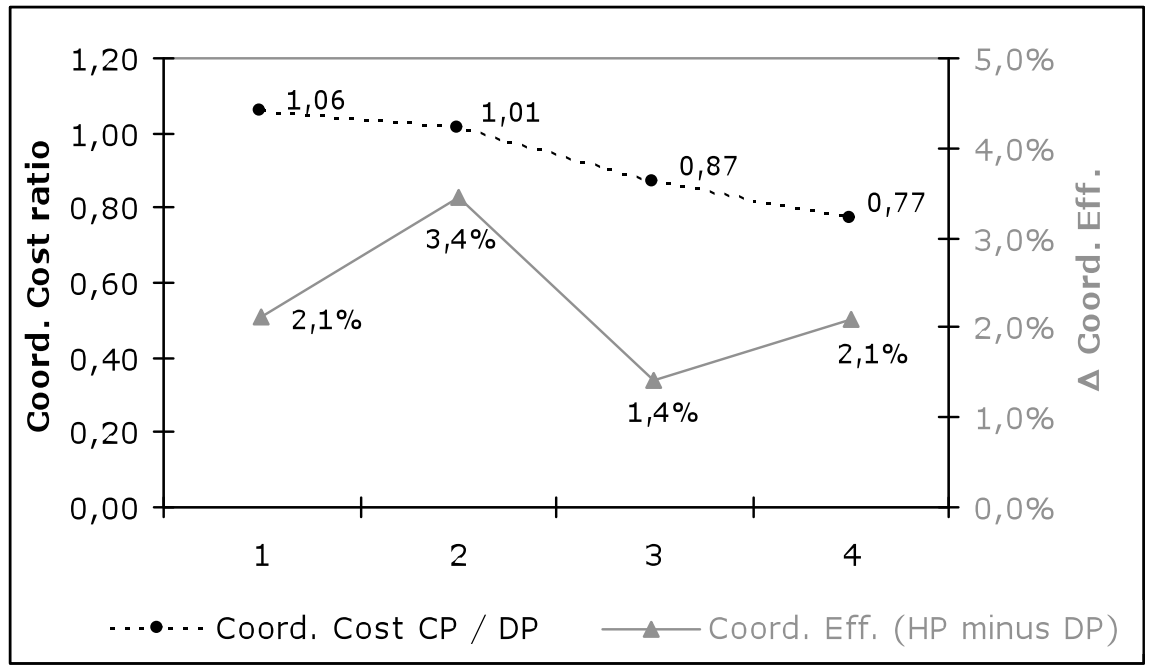

Figure 6. "Coordination cost and coordination efficiency of different coordination forms". 
For practitioners addressing SCM, some generic guidelines are as provided based on the previous findings: If the network runs with overcapacity, central planning (DP or HP) is not likely to improve coordination benefits enough to outweigh the associated cost. Otherwise, if capacity constraints are an important issue, central planning is recommendable. If machine availability is relatively uncertain, the DP type is probably a better option than HP. The implementation of a demand planning IT tool (in case of HP) is most efficient if the operation level is quite predictable.

The current investigation reveals how different organization forms can be evaluated. In order to quantify total coordination efficiency, three concepts are distinguished: coordination benefits, structural and activity-dependent costs. The latter cost category is only relevant, if capacity constraints are violated so that an iterative planning procedure is triggered. This is only the case if a static view reveals that demand constantly exceeds capacity or if in a dynamic environment a temporarily demand-capacity disequilibrium occurs. The problem of constantly insufficient capacities can rarely be solved by mid-term production planning. In contrast, under conditions of stochastic demand and availability of capacity, the probability of iterative planning varies widely as a function of the uncertainty levels. The efficiency of coordination forms then depends on the number of iterations necessary. To quantify the effect, simulation is required. Nevertheless, concerning APS, in the present investigation only the use of a demand planning module was evaluated. Its benefit was assumed to be associated with a $50 \%$ reduction of forecast errors. In fact, the real improvement depends on various influence factors, such as distribution organization, product and market characteristics, etc. Therefore the model should be expanded in this sense. With respect to inferior (production) planning levels, the evaluation of a planning optimizer would be of particular interest. This means that the efficiency of simple planning heuristics applied in the present simulation would be compared with an optimized production plan.

\section{References}

Beamon, B. M., \& Chen, V.C.P. (2001). Performance analysis of conjoined supply chains. International Journal of Production Research, 39(14), 3195-3218.

Garavelli, A. C. (2003). Flexibility configurations for the supply chain management. International Journal of Production Economics, 85, 141-153. 
Gupta, A., Maranas, C. D. (2003). Managing demand uncertainty in supply chain planning. Computers and Chemical Engineering, 27, 1219-1227.

Kim, S.W., \& Park, S. (2008). Development of a three-echelon SC model to optimize coordination costs. European Journal of Operational Research, 184(3), 1044-1061.

Lassen, S. (2006). Harmonisierung von ERP-/PPS-Prozessen und -Systemen. In: G. Schuh (Ed.), Produktionsplanung und-steuerung. (p. 377). Heidelberg: Springer.

Li, X., Wang, Q. (2007). Coordination mechanism of supply chain systems. European Journal of Operational Research, 179 (1), 1-16.

Meijboom, B., Obel, B. (2007). Tactical coordination in a multi-location and multistage operations structure: A model and a pharmaceutical company case. Omega, $35,258-273$.

Roesgen, R., \& Schuh, G. (2006): Grundlagen der Produktionsplanung und steuerung, In: G. Schuh (Ed.), Produktionsplanung und -steuerung (p. 377). Heidelberg: Springer.

Schneeweiss, C., \& Zimmer, K. (2004). Hierarchical coordination mechanisms within the supply chain. European Journal of Operational Research, 153, 687-703.

Schmenner, R.W. (1982). Multiplant manufacturing strategies among the Fortune 500. Journal of Operations Management. 2(2) (77-86).

Schotten, M. (1998). Beurteilung EDV-gestützter Koordinationsinstrumentarien in der Fertigung. Aachen: Shaker.

Stadtler, H., \& Kilger, C. (2000). Supply Chain Management and Advanced Planning (p. IX). Heidelberg: Springer. 
Article's contents are provided on a Attribution-Non Commercial 3.0 Creative commons license. Readers are allowed to copy, distribute and communicate article's contents, provided the author's and Journal of Industrial Engineering and Management's names are included. It must not be used for commercial purposes. To see the complete license contents, please visit http://creativecommons.org/licenses/by-nc/3.0/. 